(Supporting Information)

\title{
A Dinuclear Ru(II) Chelateing Amido Complex: Synthesis, Characterization, and Coupling Reaction with Carbon Monoxide
}

\author{
Shin Takemoto, Shinya Oshio, Hiroyuki Matsuzaka, * Masatsugu Hoshi, Hironobu Okimura, \\ Masayo Yamashita, Hitoshi Miyasaka, Tomohiko Ishii, and Masahiro Yamashita
}

Department of Chemistry, Faculty of Arts and Sciences, Osaka Prefecture University, Sakai, Osaka 599-8531, Japan, Coordination Chemistry Laboratories, Institute for Molecular Science, Myodaiji, Okazaki 444-8585, Japan, and Department of Chemistry, Graduate School of Science, Tokyo Metropolitan University, Hachioji, Tokyo 192-0397, Japan

\section{Contents}

Experimental Procedure

Table S1. Crystallographic Data for $\mathbf{1 b}, \mathbf{4} \cdot\left(\mathrm{CH}_{2} \mathrm{Cl}_{2}\right)_{2}$, and $\mathbf{5} \cdot \mathbf{M e C N}$.

Figure S1. ORTEP drawing of $\mathbf{1 b}$.

Figure S2. ORTEP drawing of 4 .

Figure S3. ORTEP drawing of 5. 


\section{Experimental Procedure}

General. All manipulations were carried out under an atmosphere of dinitrogen using standard Schlenk-line techniques unless otherwise mentioned. Solvents were dried and distilled over an appropriate drying agent under an atmosphere of dinitrogen. $[\mathrm{Cp} * \mathrm{RuCl}]_{4}$ was prepared according to

the literature. ${ }^{1}$ All commercially available reagents were used as received. NMR spectra were recorded on a JEOL ECP500 spectrometer. Infrared spectra were recorded on a Hitachi Nicolet I5040 FT-IR spectrometer. Elemental analyses were performed using a Perkin Elmer CHNS series II microanalyzer.

$\left[(\mathbf{C p} * \mathbf{R u})_{2}\left\{\mu_{2}-(\mathbf{N H})_{2} \mathbf{C}_{\mathbf{1 0}} \mathbf{H}_{6}\right\}\right]$ (1b). A solution of $n$-BuLi in $n$-hexane $(2.6 \mathrm{M}, 480 \mu \mathrm{L}, 1.26 \mathrm{mmol})$ was added to a solution of 2,3-naphthalenediamine (100 mg, $0.63 \mathrm{mmol})$ in $\mathrm{THF}(15 \mathrm{~mL})$ at $-80{ }^{\circ} \mathrm{C}$, and the mixture was stirred at $-80^{\circ} \mathrm{C}$ for $0.5 \mathrm{~h}$. To this solution was added $[\mathrm{Cp} * \mathrm{RuCl}]_{4}(342 \mathrm{mg}, 0.31$ $\mathrm{mmol}$ ) and the reaction mixture was allowed to warm to room temperature with stirring over $12 \mathrm{~h}$ to form a dark blue solution. The solvents were removed in vacuo and the residue was extracted with toluene $(30 \mathrm{~mL})$. Evaporation of toluene gave a dark blue solid, which was recrystallized from diethyl ether $(18 \mathrm{~mL})$ at $-25^{\circ} \mathrm{C}$ to give $\mathbf{1 b}$ as dark blue plates. Yield $168 \mathrm{mg}, 43 \%$. Anal. Calcd for $\mathrm{C}_{30} \mathrm{H}_{38} \mathrm{~N}_{2} \mathrm{Ru}_{2}: \mathrm{C}, 57.31 ; \mathrm{H}, 6.09 ; \mathrm{N}, 4.46$. Found: C, 56.76; H, 6.08; N, 4.56. ${ }^{1} \mathrm{H} \mathrm{NMR}(500 \mathrm{MHz}$, $\left.\mathrm{C}_{6} \mathrm{D}_{6}\right): \delta 7.53(\mathrm{~m}, 2 \mathrm{H}, \operatorname{aryl}), 7.13(\mathrm{~m}, 2 \mathrm{H}, \operatorname{aryl}), 7.11(\mathrm{~s}, 2 \mathrm{H}, \operatorname{aryl}), 4.73(\mathrm{~s}, 2 \mathrm{H}, \mathrm{NH}), 1.75(\mathrm{~s}, 15 \mathrm{H}$, Cp*). ${ }^{13} \mathrm{C}\left\{{ }^{1} \mathrm{H}\right\}$ NMR $\left(125.6 \mathrm{MHz}\right.$, toluene- $\left.d_{8}\right): \delta$ 130.8, 126.8, 122.2, 120.9, 111.4 (aryl), 75.5 $\left(C_{5} \mathrm{Me}_{5}\right), 11.7\left(\mathrm{C}_{5} M e_{5}\right)$.

$\left[(\mathbf{C p} * \mathbf{R u})_{2}\left(\mu_{2}-\mathbf{C O}\right)\left\{\mu_{2}-\mathbf{2 , 3}-(\mathbf{N H})_{2} \mathbf{C}_{\mathbf{1 0}} \mathbf{H}_{6}\right\}\right] \mathbf{( 2 \mathbf { b } ) .}$ To a solution of $\mathbf{1 b}(62 \mathrm{mg}, 0.099 \mathrm{mmol})$ in THF (5 $\mathrm{mL})$ was added $\mathrm{CO}(3.8 \mathrm{~mL}, 0.15 \mathrm{mmol})$ at room temperature and the solution was stirred for $23 \mathrm{~h}$. The solvent was removed in vacuo and the remaining solid was washed with diethyl ether (10 mL) to give $\mathbf{2 b}$ as a brown solid. Yield $37 \mathrm{mg}, 57 \%$. The product was recrystallized from toluene-hexanes to give analytically pure material. Anal. Calcd for $\mathrm{C}_{31} \mathrm{H}_{38} \mathrm{~N}_{2} \mathrm{ORu}_{2}: \mathrm{C}, 56.69 ; \mathrm{H}, 5.83 ; \mathrm{N}, 4.27$. Found: 
$\mathrm{C}, 56.80 ; \mathrm{H}, 6.00 ; \mathrm{N}, 4.29 .{ }^{1} \mathrm{H}$ NMR (500 MHz, $\left.\mathrm{C}_{6} \mathrm{D}_{6}\right): \delta 7.28(\mathrm{~m}, 2 \mathrm{H}, \operatorname{aryl}), 6.99(\mathrm{~m}, 2 \mathrm{H}, \operatorname{aryl}), 6.65$ (s, 2H, aryl), $3.86(\mathrm{~s}, 2 \mathrm{H}, \mathrm{NH}), 1.67$ (s, 30H, $\left.\mathrm{Cp}^{*}\right) . \mathrm{IR}(\mathrm{KBr}): 1763 \mathrm{~cm}^{-1}(\mathrm{v}(\mathrm{CO}))$.

$\left[\mathbf{C p} * \mathbf{R u}\left(\mu_{2}-\mathbf{C O}\right)\left\{\mu_{2}-2,3-(\mathbf{C O N H})(\mathbf{N H}) \mathbf{C}_{10} \mathbf{H}_{6}\right\} \mathbf{R u C p} *(\mathbf{C O})\right]$ (4). Into a solution of $\mathbf{1 b}$ (327 mg, 0.52 $\mathrm{mmol})$ in THF $(10 \mathrm{~mL})$ was bubbled $\mathrm{CO}$ at room temperature for $15 \mathrm{~min}$. After stirring overnight, the reaction solution was loaded onto a column of activated alumina. Elution of THF afforded a red band which was collected and evaporated to dryness. Recrystallization of the residue from $\mathrm{CH}_{2} \mathrm{Cl}_{2}$-hexanes afforded 4 as dark red crystals. Yield $273 \mathrm{mg}, 74 \%$. Anal. Calcd for $\mathrm{C}_{33} \mathrm{H}_{38} \mathrm{~N}_{2} \mathrm{O}_{3} \mathrm{Ru}_{2}$ : C, 55.61; H, 5.37; N, 3.93. Found: C, 55.53; H, 5.38; N, 3.81. ${ }^{1} \mathrm{H}$ NMR (500 MHz, $\left.\mathrm{CDCl}_{3}\right): \delta 7.70(\mathrm{~d}$, 1H, aryl), $7.67(\mathrm{~s}, 1 \mathrm{H}, \operatorname{aryl}), 7.56(\mathrm{~s}, 1 \mathrm{H}, \operatorname{aryl}), 7.28(\mathrm{~m}, 1 \mathrm{H}, \operatorname{aryl}), 7.16(\mathrm{~s}, 1 \mathrm{H}, \mathrm{NH}), 6.81(\mathrm{~s}, 1 \mathrm{H}, \operatorname{aryl})$, 6.80 (s, 1H, NH), 1.68, 1.49 (s, 15H each, Cp*). IR (nujol): 1919, 1819, $1514 \mathrm{~cm}^{-1}(\mathrm{v}(\mathrm{CO}))$.

$\left[\mathbf{C p} * \mathbf{R u}\left(\mu_{2}-\mathbf{C N B u}^{t}\right)\left\{\mu_{2}-\mathbf{2 , 3}-(\mathbf{N H})_{2} \mathbf{C}_{10} \mathbf{H}_{6}\right\} \mathbf{R u C p} *\left(\mathrm{CNBu}^{t}\right)\right]$ (5). To a solution of $\mathbf{1 b}(201 \mathrm{mg}, 0.32$ mmol) in THF ( $8 \mathrm{~mL})$ was added $t$-butyl isocyanide $(90 \mu \mathrm{L}, 0.8 \mathrm{mmol})$, and the brown solution was stirred at room temperature for $14 \mathrm{~h}$. The solution was concentrated in vacuo to $1 \mathrm{~mL}$ and layered with acetonitrile ( $10 \mathrm{~mL}$ ) to give 5 as brown microcrystalline solid. Yield $117 \mathrm{mg}, 46 \%$. The product was recrystallized from acetonitrile at $-25{ }^{\circ} \mathrm{C}$ to give analytically pure material. Anal. Calcd for $\mathrm{C}_{40} \mathrm{H}_{56} \mathrm{~N}_{4} \mathrm{Ru}_{2} \cdot \mathrm{MeCN}$ : C, 60.33; H, 7.11; N, 8.38. Found: C, 60.54; H, 7.20; N, 8.25. The presence of the solvating acetonitrile was confirmed by X-ray crystallography. ${ }^{1} \mathrm{H}$ NMR $\left(500 \mathrm{MHz}, \mathrm{C}_{6} \mathrm{D}_{6}\right): \delta 7.73$ (d, 1H aryl), $7.48(\mathrm{~d}, 1 \mathrm{H}, \operatorname{aryl}), 7.32(\mathrm{~s}, 1 \mathrm{H}, \operatorname{aryl}), 7.30(\mathrm{t}, 1 \mathrm{H}, \operatorname{aryl}), 7.05(\mathrm{t}, 1 \mathrm{H}, \operatorname{aryl}), 6.91(\mathrm{br} \mathrm{s}, 1 \mathrm{H}$, NH), 6.34 (s, 1H, aryl), 1.81 (br s, 1H, NH), 1.71, 1.68 (s, 15H each, Cp*), 1.49, 0.80 (s, 9H each, $t$ $\mathrm{Bu})$. IR (hexane): $2105,2074,1775 \mathrm{~cm}^{-1}(\mathrm{v}(\mathrm{CN}))$.

X-ray Crystallography. Crystallographic data are summarized in Table S1. A single crystal suitable for X-ray crystallography was sealed in a grass capillary. All measurements were performed on a Rigaku RAXIS Rapid diffractometer equipped with an imaging plate detector. The frame data 
were processed using the Rigaku PROCESS-AUTO program, ${ }^{2}$ and the reflection data were corrected for absorption with an Abscor program. ${ }^{3}$

The structures were solved by direct method and refined on $F^{2}$ by full-matrix least-squares method by using SHELX97. ${ }^{4}$ Anisotropic refinement was applied to all non hydrogen atoms, and hydrogen atoms were located at the caluculated positions and treated as riding models.

\section{References}

(1) Fagan, P. J.; Ward, M. D.; Calabrese, J. C. J. Am. Chem. Soc. 1989, 111, 1698.

(2) PROCESS-AUTO, Automatic Data Acquisition and Processing Package for Imaging Plate Diffractometer; Rigaku Corporation: Tokyo, Japan, 1998.

(3) Higashi, T. ABSCOR, Empirical Absorption Correction based on Fourier Series Approximation; Rigaku Corporation: Tokyo, Japan, 1995.

(4) Sheldrick, G. M. SHELX97, Program for Crystal Structure Determination; University of Göttingen: Göttingen, Germany, 1997. 
Table S1. Crystallographic data for $1 \mathrm{~b}, 4 \cdot\left(\mathrm{CH}_{2} \mathrm{Cl}_{2}\right)_{2}$, and $5 \cdot \mathrm{MeCN}$.

\begin{tabular}{|c|c|c|c|}
\hline & $1 \mathrm{~b}$ & $4 \cdot\left(\mathrm{CH}_{2} \mathrm{Cl}_{2}\right)_{2}$ & $5 \cdot \mathrm{MeCN}$ \\
\hline formula & $\mathrm{C}_{30} \mathrm{H}_{38} \mathrm{~N}_{2} \mathrm{Ru}_{2}$ & $\mathrm{C}_{35} \mathrm{H}_{42} \mathrm{~N}_{2} \mathrm{O}_{3} \mathrm{Cl}_{4} \mathrm{Ru}_{2}$ & $\mathrm{C}_{42} \mathrm{H}_{59} \mathrm{~N}_{5} \mathrm{Ru}_{2}$ \\
\hline$M$ & 628.76 & 882.65 & 836.08 \\
\hline$T / \mathrm{K}$ & 296 & 296 & 296 \\
\hline crystal size (mm) & $0.60 \times 0.40 \times 0.20$ & $0.80 \times 0.50 \times 0.30$ & $0.30 \times 0.20 \times 0.10$ \\
\hline crystal system & monoclinic & triclinic & monoclinic \\
\hline space group & $P 2_{1} / c$ & $P 1$ & $P 2_{1} / c$ \\
\hline$Z$ & 4 & 2 & 4 \\
\hline$a(\AA)$ & $11.483(15)$ & $11.634(5)$ & $11.510(3)$ \\
\hline$b(\AA)$ & $15.91(2)$ & $12.399(5)$ & $16.845(5)$ \\
\hline$c(\AA)$ & $16.02(2)$ & $13.935(8)$ & $22.298(7)$ \\
\hline$\alpha(\operatorname{deg})$ & 90 & $105.730(18)$ & 90 \\
\hline$\beta(\operatorname{deg})$ & 105.74(10) & $100.04(2)$ & $105.30(2)$ \\
\hline$\gamma(\operatorname{deg})$ & 90 & $95.60(2)$ & 90 \\
\hline$V\left(\AA^{3}\right)$ & $2817(6)$ & $1882.8(15)$ & 4170(2) \\
\hline$D_{\text {calc }}\left(\mathrm{g} / \mathrm{cm}^{3}\right)$ & 1.483 & 1.557 & 1.332 \\
\hline$\mu(\mathrm{Mo}-\mathrm{K} \alpha)\left(\mathrm{mm}^{-1}\right)$ & 1.092 & 1.121 & 1.040 \\
\hline reflections collected & 21148 & 18159 & 40012 \\
\hline unique reflections & 4884 & 8259 & 9535 \\
\hline GOF on F2 & 1.076 & 1.034 & 1.068 \\
\hline $\mathrm{R} 1[I>2 \sigma(I)]^{a}$ & 0.1406 & 0.0414 & 0.0399 \\
\hline wR2 (all data) ${ }^{b}$ & 0.3696 & 0.1146 & 0.0993 \\
\hline
\end{tabular}


Figure S1. ORTEP drawing of $1 \mathrm{~b}$.

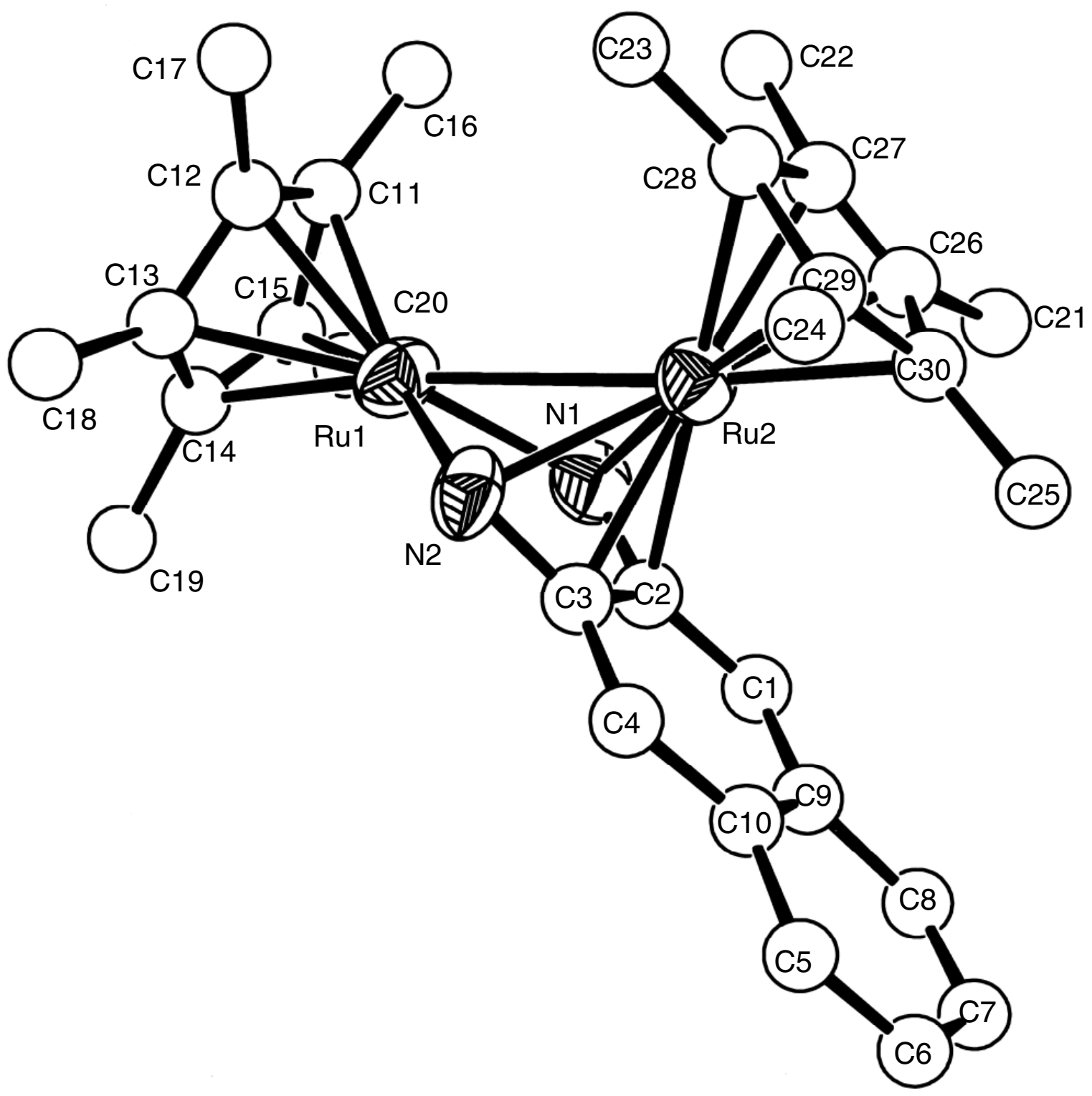


Figure S2. ORTEP drawing of 4.

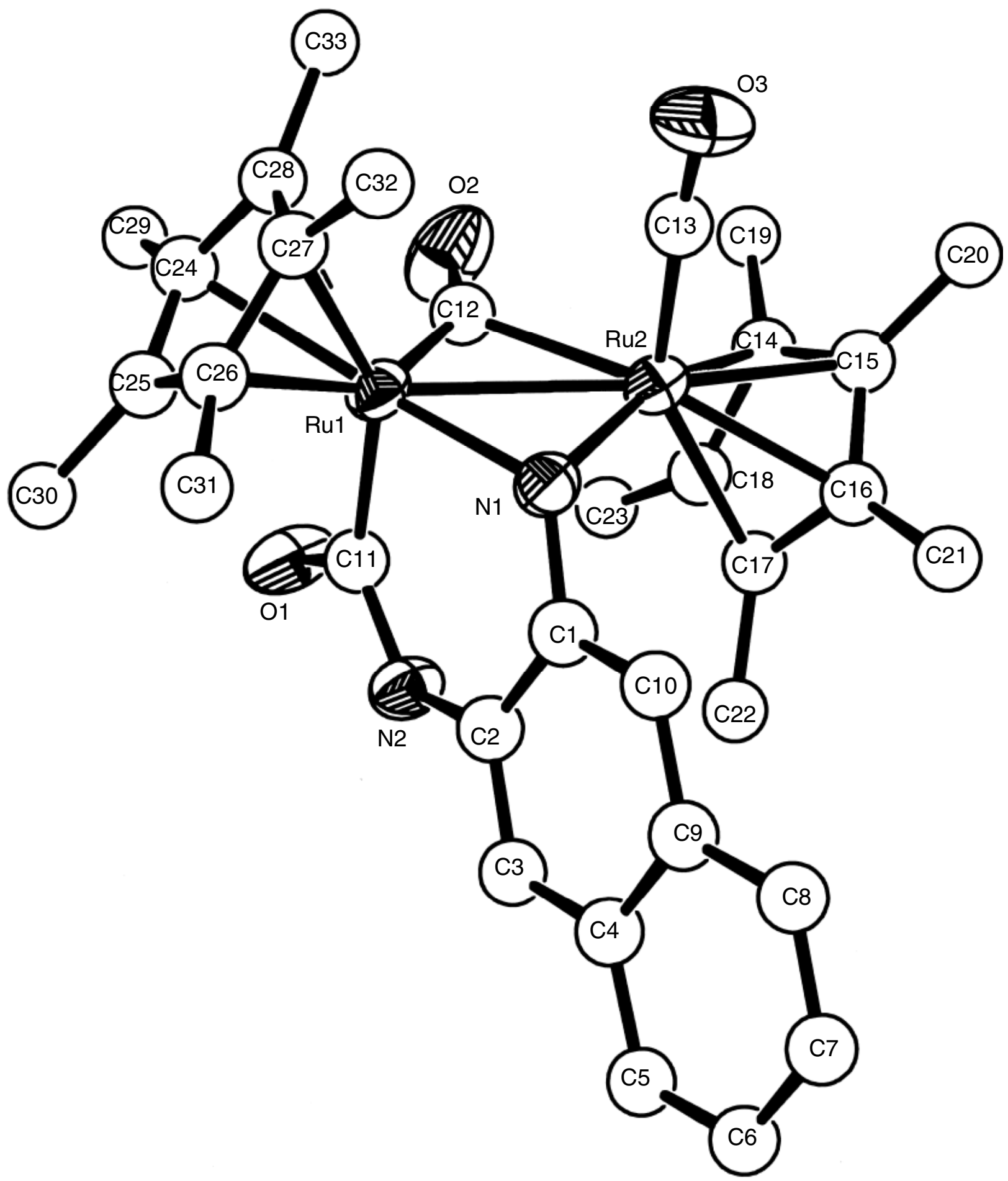


Figure S3. ORTEP drawing of 5.

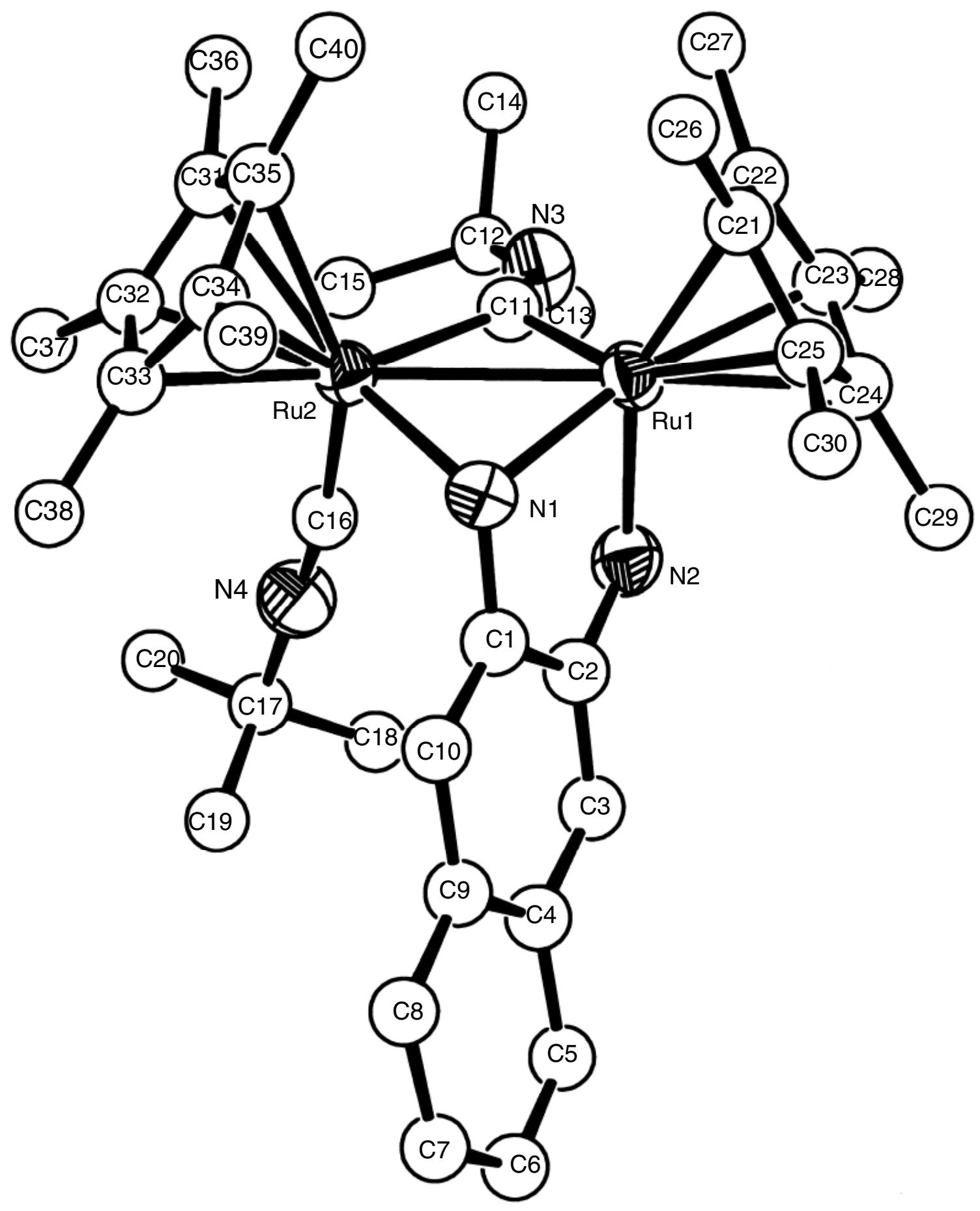

